\title{
Fexinidazole and Human African Trypanosomiasis: good news for this important neglected tropical disease
}

\author{
Taciana de Souza Bayão ${ }^{[1]}$, Marli do Carmo Cupertino ${ }^{[1],[2],}$ \\ Andréia Patrícia Gomes ${ }^{[1]}$ and Rodrigo Siqueira-Batista ${ }^{[1],[2]}$
}

[1]. Universidade Federal de Viçosa, Departamento de Medicina e Enfermagem, Programa de Pós-graduação em Ciências da Saúde, Viçosa, MG, Brasil.

[2]. Faculdade Dinâmica do Vale do Piranga, Escola de Medicina, Ponte Nova, MG, Brasil.

\section{Dear Editor:}

Human African trypanosomiasis (HAT), or sleeping sickness, is an endemic disease in 36 sub-Saharan African countries, typically occurring in underdeveloped areas, where health systems face significant difficulties of diverse natures. The disease is caused by the protozoa of the species Trypanosoma brucei gambiense (98\% of cases) and Trypanosoma brucei rhodesiense, transmitted via the bite of the tse-tsé fly (genus Glossina) ${ }^{1}$. Since the 20th century, three major epidemics have been reported in sub-Saharan Africa ${ }^{2}$. According to data from the World Health Organization (WHO), it is estimated that, currently, there are 20,000 cases, and about 65 million people are at risk of being infected ${ }^{3}$. The disease is usually fatal if no adequate diagnosis and treatment are available due to the multiplication of the parasites in the human body, with the subsequent invasion of the central nervous system. Mortality is primarily caused by the difficulties in follow-up, due to the complexity of diagnosis and therapy and the need for specialized professionals $\mathrm{s}^{3,4,5}$.

The type of treatment depends on the stage of the disease. Clinically HAT occurs in two stages. The first, i.e., the hemolymphatic stage, is characterized by unspecific clinical signs such as fever, asthenia, and peripheral edema. In the second stage, i.e., the meningoencephalic stage, rupture of the

Corresponding author: Rodrigo Siqueira-Batista.

e-mail: rsiqueirabatista@yahoo.com.br

Orcid: 0000-0002-3661-1570

Received 27 April 2019

Accepted 18 July 2019 blood-brain barrier and migration of the protozoa to the brain can occur, triggering a neurological picture with seizures, psychological and cognitive alterations, and sleep disorders; this may ultimately progress to death ${ }^{4}$. The earlier the disease is identified, the better the prospect of cure, as successful treatment in the second stage is dependent on drugs that cross the bloodbrain barrier to reach the parasite. Moreover, these drugs are more toxic and difficult to administer than those utilized in the first stage $e^{3,45}$.

From a historical perspective, therapeutic options for the disease at the beginning of the $21^{\text {st }}$ century were still less than that expected for accessible and effective treatment. In total, five different drugs were used and all showed considerable toxicity, were costly, and were required to be administered parenterally ${ }^{2,5}$. The therapeutic approach of HAT in the first stage was possible only with intramuscular injections of pentamidine for T. b. gambiense and suramin for T. b. rhodesiense ${ }^{2}$. In the second stage of the disease, diagnosed by cerebrospinal fluid analysis, the drug melarsoprol was the first choice for gambiense HAT and the only option for the Rhodesian disease $\mathrm{e}^{2,5}$. However, the high toxicity of melarsoprol, estimated as approximately 3 to $10 \%$ in users and typically evolving to a fatal outcome $\mathrm{e}^{3,6}$, resulted in the drug eflornithine, associated with nifurtimox, to gradually gain space in the treatment of T. $b$. gambiense, in endemic areas ${ }^{6}$. The nifurtimox-eflornithine (NECT) option had been shown to be clinically more interesting. However, some disadvantages still persisted: high costs, logistic and infrastructural difficulties faced in rural clinics with regard to the administration of daily intravenous infusions for at least two weeks, and the need for invasive procedures for curative control ${ }^{2,7,8}$. The need for investment in the study and development of other potential 
therapies, coupled with the significant efforts of the Drugs for Neglected Diseases Initiative (DNDi) in partnership with other entities, culminated in an oral formulation of fexinidazole ${ }^{3}$.

By the end of 2018, a major advance in the treatment of the disease was achieved, with a positive opinion of the Committee for Medicinal Products for Human Use (CHMP) being published in Article 58 of Regulation (EC) No 726/2004 1 , for the use of a new drug called fexinidazole ${ }^{7}$. This drug, developed by the DNDi in partnership with Sanofi, is a major venture as a tool to eradicate the disease, WHO's goal for $2020^{3,6}$. The drug achieved a $91.2 \%$ success rate in the late-stage of the T. b. gambiense disease, 18 months after treatment, in a clinical trial in the Democratic Republic of Congo and the Central African Republic?. This drug is the product of a series of high investments in research using molecular and biochemical approaches, as well as laboratory and clinical trials 5 . Fexinidazole is a derivative of 5-nitroimidazole, which acts as an inhibitor of protozoal DNA synthesis. It is the first exclusively oral treatment, proved to be effective at both stages of the disease ${ }^{4}$. The first-choice NECT therapy showed a $98 \%$ success rate in the same study; however, it needs to be administered intravenously in a hospital environment by trained professionals 9

In in vitro assays, fexinidazole was reported to be less cytotoxic compared with melarsoprol ${ }^{10}$. In in vivo assays, it has been demonstrated that the oral formulation administered in rats has a high potential for cure in the acute and chronic stages of the disease, owing to the good absorption and distribution profile in the body, including in regions of the brain ${ }^{8,11}$. Phase I trials in humans, initiated after compliance with international regulatory guidelines regarding preclinical experiments, have indicated the viability of the $\operatorname{drug}^{6}$. Therefore, phase II and III studies, published in 2018, presented fexinidazole as a possible alternative to NECT in individuals aged 15 years or older. These clinical trials were also useful in estimating drug safety and delimiting the current recommendation to patients with a minimum age of six years and weighing more than $20 \mathrm{~kg}^{7,9}$.

Thus, the pharmacological and therapeutic advantages demonstrated by fexinidazole have significant social impacts due to the possibility of an oral formulation with high efficacy and low drug toxicity, together with good clinical results and lower side effects. These factors allow the expansion of access to economically disadvantaged populations, even in the absence of an adequate health care structure and without the need for specialized personnel, collectively ensuring higher efficiency in the eradication of the disease ${ }^{3,9}$. This therapy, in most cases, is expected to result in a significant cure in individuals at both the hemolymphatic and meningoencephalic stages of the disease, as well as a significant reduction in hospital admissions and lumbar punctures ${ }^{1,2,6.12}$.

Positive evaluation by the CHMP has triggered considerable impacts, such as the start of commercialization in the endemic countries from 2019 and the prospect of Sanofi donations in partnership with WHO for free distribution in the underdeveloped regions ${ }^{7}$. Such a possibility brings a new tool that can simplify and facilitate case management and contribute to achieving the goal of eliminating sleeping sickness as a public health problem. The next steps involve the need to ensure the timely delivery of the drug, together with the appropriate training of healthcare professionals in the efficacious use of fexinidazole ${ }^{3,9}$. Directing the treatment of HAT in an accessible and practicable way to the primary level of health care is perceived to be a rational approach in the treatment of neglected diseases. In this perspective, we emphasize that the joint efforts of political, technological, scientific, and humanitarian support are promising paths that deserve to be extended to other neglected tropical diseases, such as Chagas disease and leishmaniasis.

\section{ACKNOWLEDGMENTS}

This study was supported by the Conselho Nacional de Desenvolvimento Científico e Tecnológico (CNPq - National Council for Scientific and Technological Development) and the Research Program of the Faculdade Dinâmica do Vale do Piranga (PROAPP/FADIP).

\section{Conflict of Interest}

The authors declare that there is no conflict of interest.

Financial Support

CNPq and PROAPP/FADIP.

\section{REFERENCES}

1. Bottieau E, Clerinx J. Human African Trypanosomiasis: Progress and Stagnation. Infectious Disease Clinics. 2019;33(1):61-77.

2. Brun R, Don R, Jacobs RT, Wang MZ, Barrett MP. Development of novel drugs for human African trypanosomiasis. Future Microbiol. 2011;6(6):677-91.

3. Franco JR, Cecchi G, Priotto G, Paone M, Diarra A, Grout L, et al. Monitoring the elimination of human African trypanosomiasis: Update to 2016. PLoS Negl Trop Dis . 2018; 12(12): e0006890.

4. Pollastri MP. Fexinidazole: A new drug for african sleeping sickness on the horizon. Trends Parasitol. 2018;34(3):178-9.

5. Burri C. Chemotherapy against human African trypanosomiasis: is there a road to success? Parasitology. 2010;137(14):1987-94.

6. Tarral A, Blesson S, Mordt OV, Torreele E, Sassella D, Bray MA, et al. Determination of an optimal dosing regimen for fexinidazole, a novel oral drug for the treatment of human African trypanosomiasis: first-in-human studies. Clin Pharmacokinet. 2014;53(6):565-80.

7. Deeks ED. Fexinidazole: First global approval. Drugs. 2019;79(2):215-20.

8. Torreele E, Bourdin Trunz B, Tweats D, Kaiser M, Brun R, Mazué $\mathrm{G}$, et al. Fexinidazole-a new oral nitroimidazole drug candidate entering clinical development for the treatment of sleeping sickness. PLoS neglected tropical diseases. 2010; 4(12): e923.

9. Mesu VKBK, Kalonji WM, Bardonneau C, Mordt OV, Blesson S, Simon F, et al. Oral fexinidazole for late-stage African Trypanosoma brucei gambiense trypanosomiasis: a pivotal multicentre, randomised, non-inferiority trial. The Lancet. 2018;391(10116):144-54.

10. Tweats D, Trunz BB, Torreele E. Genotoxicity profile of fexinidazole - A drug candidate in clinical development for human african trypanomiasis (sleeping sickness). Mutagenesis. 2012;27(5):523-32.

11. Burrell-Saward H, Harris AJ, de LaFlor R, Sallam H, Alavijeh MS, Ward TH, et al. Dose-dependent effect and pharmacokinetics of fexinidazole and its metabolites in a mouse model of human african trypanosomiasis. Int J Antimicrob Agents. 2017;50(2):203-9.

12. Chappuis F. Oral fexinidazole for human african trypanosomiasis. The Lancet. 2018;391(10116):100-2. 